\title{
Assembly Deformation and Stresses of Compliant Structures
}

\author{
Chensong Dong ${ }^{1, a}$ and Lu Kang ${ }^{1, b}$ \\ ${ }^{1}$ Department of Mechanical Engineering, Curtin University of Technology, GPO Box U1987, Perth, \\ WA 6845, Australia \\ ac.dong@curtin.edu.au, ${ }^{\mathrm{b} l u . k a n g @ s t u d e n t . c u r t i n . e d u . a u ~}$
}

Keywords: Compliant; assembly; tolerance

\begin{abstract}
Compliant components such as large sheet metal components are commonly used in various products including automotive, aircraft and home appliances. Because of part-to-part variations, deformation and stresses are induced in the assembly process. An approach to the assembly tolerance analysis of compliant structures is presented in this paper. Given component deformation, assembly deformation and stresses are derived by finite element analysis (FEA). The influence of component deformation on assembly deformation and stresses is studied by response surface methodology (RSM), and a regression model is developed. Using the developed regression model, Monte Carlo simulation was conducted to study assembly tolerance and stresses. This approach is illustrated by an example.
\end{abstract}

\section{Introduction}

Compliant components such as large sheet metal components are commonly used in various products including automotive, aircraft and home appliances. Because of part-to-part variations, deformation and stresses are induced in the assembly process.

Primary methods for analyzing variation in assemblies are worst case analysis, root sum squares and Monte Carlo simulation [1]. All these methods assume that the parts in the assembly are rigid and are not applicable to compliant structures. For analyzing the variation of compliant assemblies, finite element analysis (FEA) is usually employed [2-4]. However, because FEA requires high computation load, it is infeasible in assembly tolerance analysis.

In this paper, an approach to the assembly tolerance analysis of compliant structures is presented. Given component deformation, assembly deformation and stresses are derived by finite element analysis (FEA). The influence of component deformation on assembly deformation and stresses is studied by response surface methodology (RSM), and a regression model is developed. Using the developed regression model, Monte Carlo simulation was conducted to study assembly tolerance and stresses. This approach is illustrated by an example.

\section{Methodology}

The assembly process of compliant structures is schematically illustrated in Fig. 1. Two components are fixed at the left end and joined or welded at the right end. Because of the processinduced deformation, the right ends deviate from the nominal position. Thus, clamping forces are applied to bring them back to the nominal position. After these two parts are joined and the clamping forces are removed, because of residual stresses, the assembly deviates from the nominal position.

As shown in Fig. 1(a), in the Cartesian coordinate system, the component deformation is characterized by the angular deflections $\theta_{1}$ and $\theta_{2}$, respectively. Because of these angular deflections, the right ends deviate to locations $y_{10}$ and $y_{20}$, respectively.

In order to bring the right ends to the nominal position, clamp forces are needed, as shown in Fig. 1(b). The deformation is related to the clamping force by the force-displacement equation, i.e.

$$
F=K \delta
$$


where $F=$ clamping force; $\delta=$ displacement; and $K=$ stiffness.

Alternatively, the displacement-force equation is given by

$$
\delta=\frac{1}{K} F
$$

When clamping forces are released, as shown in Fig. 1(d), these two parts are held due to bond forces. From equilibrium, the bond forces have the following relationship.

$$
-F_{b 1}=F_{b 2}=F_{b}
$$

It is also seen that the right ends of these two parts are at the same position, i.e.

$$
y_{1}=y_{10}+\delta_{1 y}=y_{20}+\delta_{2 y}=y_{2}
$$

Combining Eqs. (1) to (4) we have

$$
y_{10}-\frac{1}{K_{1}} F_{b}=y_{20}+\frac{1}{K_{2}} F_{b}=y_{b}
$$

Solving Eq. (5) the bond force is given by

$$
F_{b}=\frac{K_{1} K_{2}\left(y_{10}-y_{20}\right)}{K_{1}+K_{2}}
$$

Substitute Eq. (6) into Eq. (5) the assembly deflection is given by

$$
y_{b}=\frac{K_{1} y_{10}+K_{2} y_{20}}{K_{1}+K_{2}}
$$

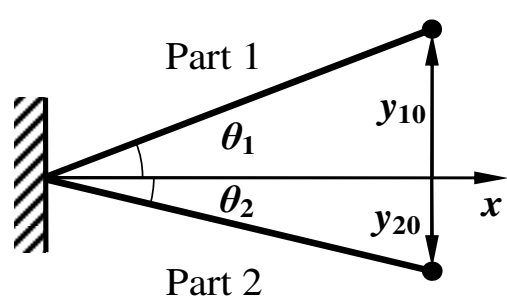

(a)

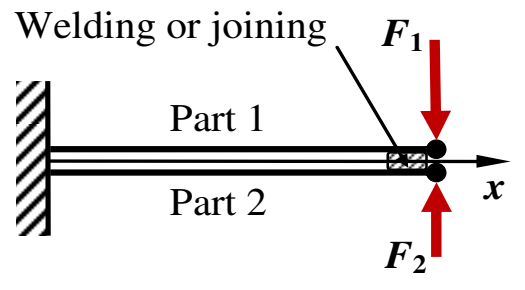

(c)

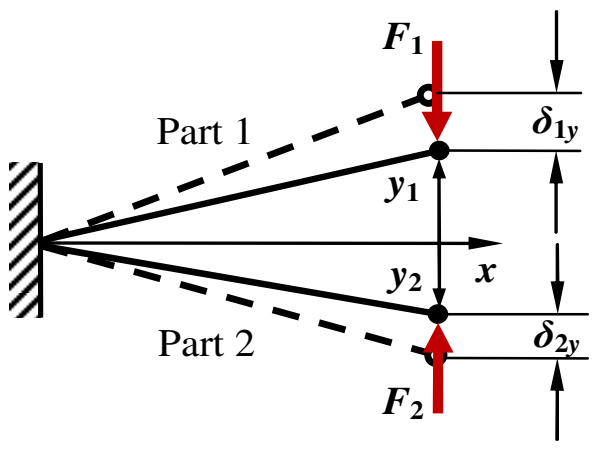

(b)

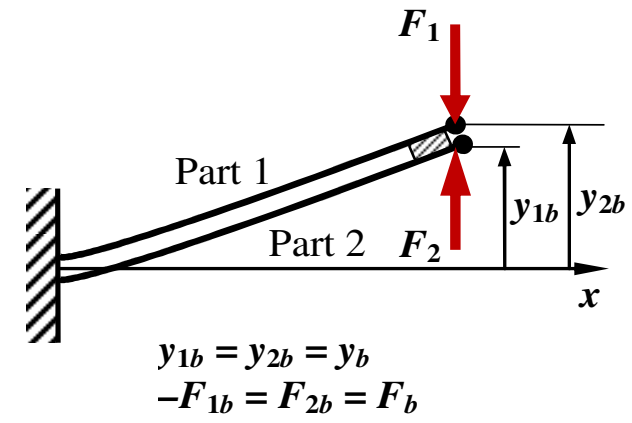

(d)

Fig. 1: Assembly process

(a) Parts deviate from nominal design; (b) Clamping force applied; (c) Welding/joining; (d) Clamp released and spring-back

For complex geometries, because closed-form solutions are difficult to found, the displacement after assembly can be found by finite element analysis. For the purpose of assembly tolerance analysis, a large number of data is needed to obtain a satisfactory distribution for assembly deformation [5]. This is usually done by using Monte Carlo methods. The direct Monte Carlo 
method is infeasible since it requires FEA simulation each time, which is tedious and timeconsuming. In this study, an alternative approach based on response surface methodology (RSM) is developed.

When RSM is used, input variables are the deformation of each component. For each variable, several levels are chosen and these levels are combined in a given way. For each combination, FEA simulation is conducted and the assembly deformation is found. In addition, the maximum residual stress is also found. Based on the results, a regression model is developed. Monte Carlo simulation can be conveniently conducted using this developed model, and the statistical distributions of assembly deformation and residual stress are found from the variation of components.

\section{Application}

In this section, the assembly of two aluminum sheet metal parts as shown in Fig. 2 was studied by the method presented in the previous section. The depth and thickness of both parts are $200 \mathrm{~mm}$ and $3 \mathrm{~mm}$, respectively. Part 1 and part 2 deviate from the nominal position due to the residual stresses. For both parts, the range of angular deflection was assumed to be 1-5 degrees. For each angular deflection, three levels 1, 3, and 5 degrees were chosen and a three-level factorial design was employed to develop the regression model. The results from FEA are shown in Table 1.
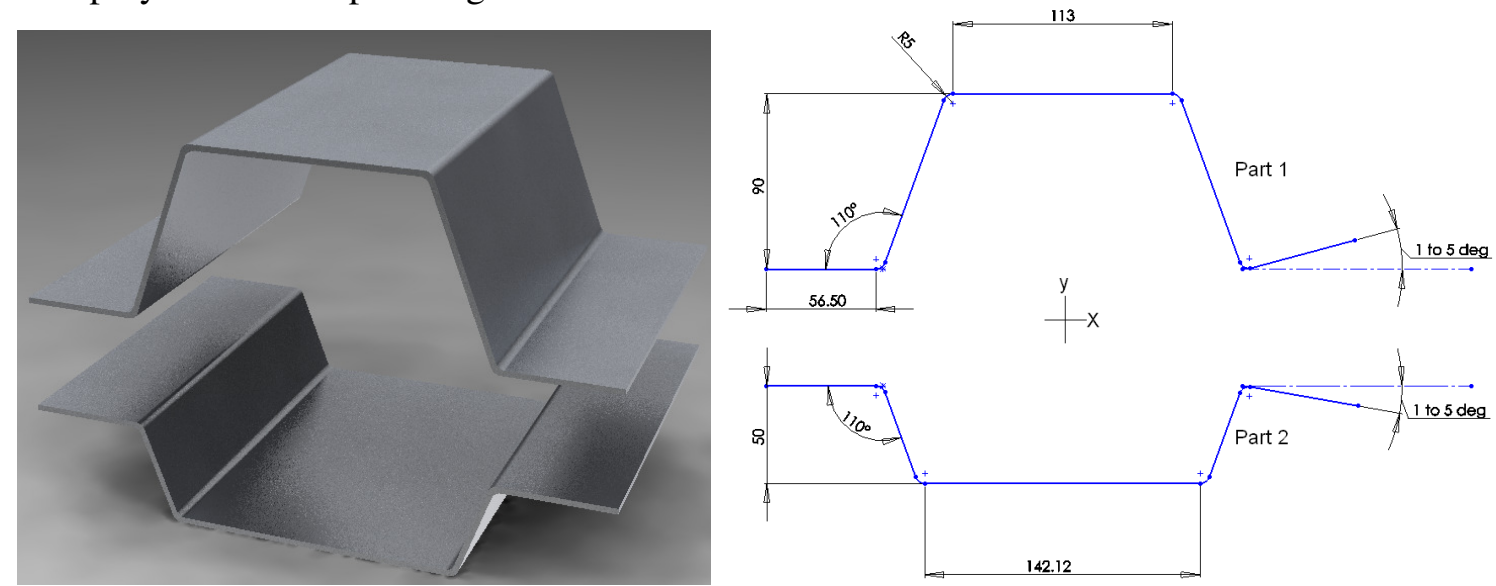

Fig. 2: Assembly of two aluminum sheet metal parts

Table 1: Three-level factorial design

\begin{tabular}{ccccc}
\hline$\theta_{1}\left(^{\circ}\right)$ & $\theta_{2}\left(^{\circ}\right)$ & $F_{b}(\mathrm{~N})$ & $y_{b}(\mathrm{~mm})$ & $\sigma_{r}(\mathrm{MPa})$ \\
\hline 1 & 1 & 0.23 & -0.07 & 2.33 \\
1 & 5 & 0.70 & -2.30 & 6.97 \\
5 & 1 & 0.70 & 1.88 & 6.97 \\
5 & 5 & 1.16 & -0.35 & 11.62 \\
3 & 3 & 0.70 & -0.21 & 6.98 \\
1 & 3 & 0.47 & -1.19 & 4.65 \\
3 & 1 & 0.47 & 0.91 & 4.65 \\
5 & 3 & 0.93 & 0.77 & 9.30 \\
3 & 5 & 0.93 & -1.32 & 9.30 \\
\hline
\end{tabular}

The regression model is fitted as

$$
\begin{aligned}
& y_{b}=-4.23 \times 10^{-5}+0.488 \theta_{1}-0.558 \theta_{2} \\
& \sigma_{r}=6.15 \times 10^{-3}+1.162\left(\theta_{1}+\theta_{2}\right)
\end{aligned}
$$

The regression model is graphically shown in Fig. 3. Monte Carlo simulation was conducted using the developed regression model. The angular deflections of these two components were assumed to be normally distributed $N(3,2 / 3)$. 100,000 random data were generated for both angles. The resulting assembly deflection and corresponding residual stress were calculated using the regression model. 
The histograms of the assembly deflection and residual stress are shown in Fig. 4. It is seen that the distribution of the assembly deflection is $N(-0.210,0.494) \mathrm{mm}$ and that of the residual stress is $N(6.98,1.09) \mathrm{MPa}$.

\section{Conclusions}

Compliant components such as large sheet metal components are commonly used in various industries. Because of part-to-part variations, deformation and stresses are induced in the assembly process. An approach to the assembly tolerance analysis of compliant structures is presented in this paper. For a given assembly, a regression model can be developed by response surface methodology (RSM) based on FEA results. The advantage is it avoids the repeated FEA, and thus saves time and cost. Assembly deformation and stresses can be convenient analyzed by Monte Carlo simulation.
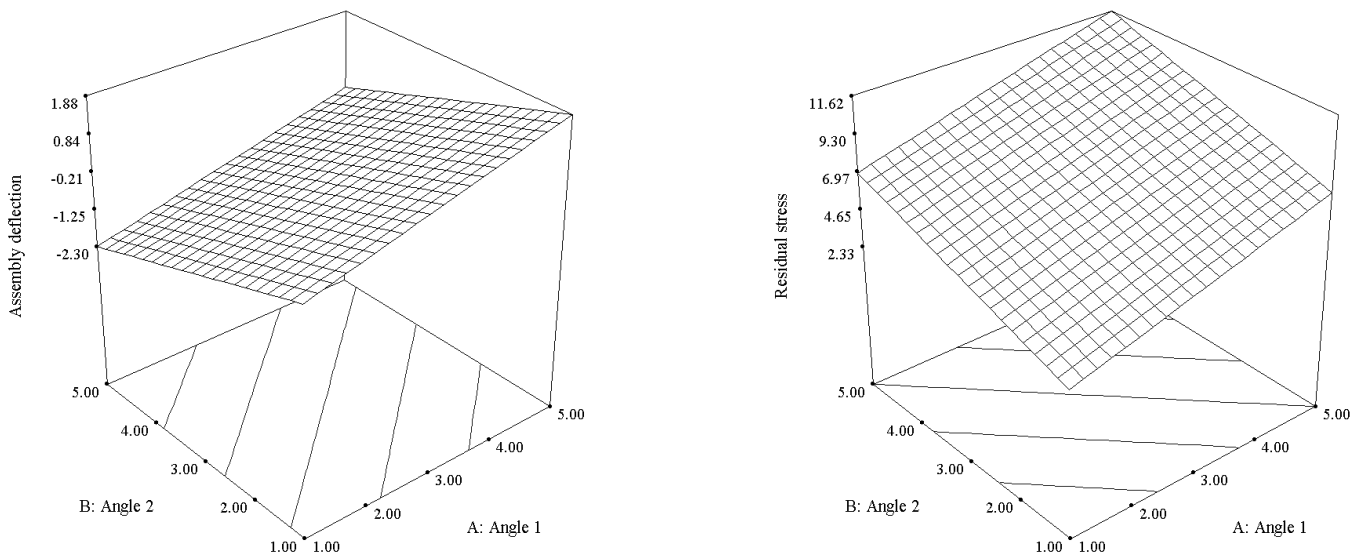

Fig. 3: Regression model (Left: assembly deflection; right: residual stress)
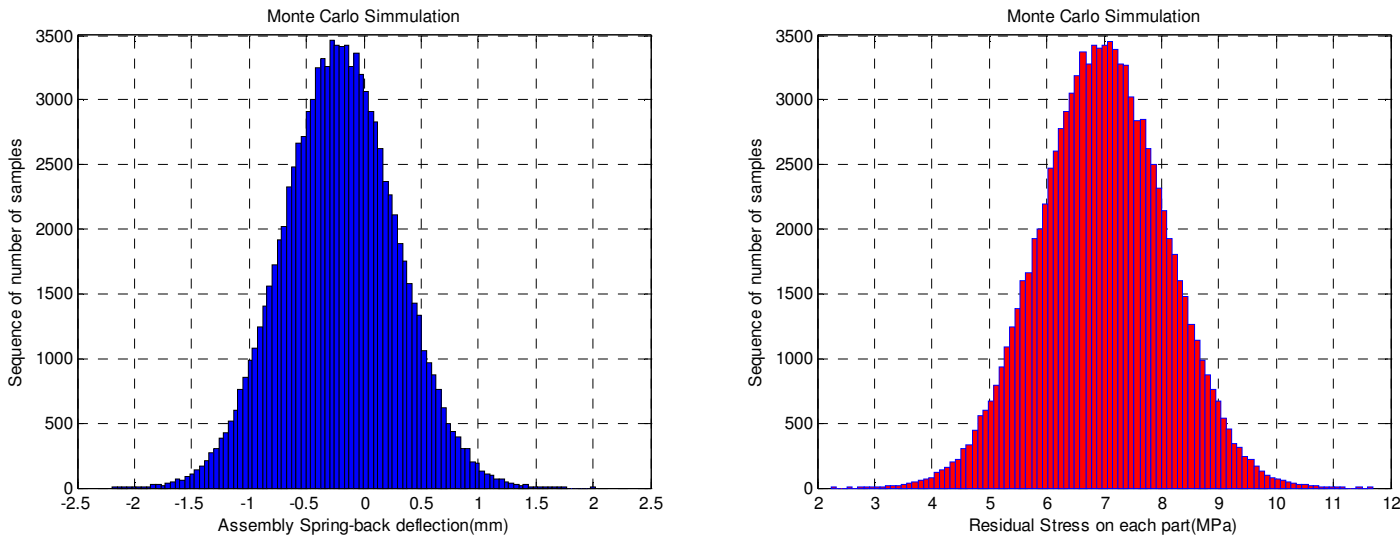

Fig. 4: Results from Monte Carlo simulation (Left: assembly deflection; right: maximum residual stress)

\section{References}

[1] K.W. Chase and A.R. Parkinson: Research in Engineering Design Vol. 3 (1991), p. 23

[2] W.W. Cai, C. Hsieh, Y. Long, S.P. Marin and K.P. Oh: ASME J. Manuf. Sci. Eng. Vol. 128 (2006), p. 270

[3] K. Xie, L. Wells, J.A. Camelio and B.D. Youn: ASME J. Manuf. Sci. Eng. Vol. 129 (2007), p. 934

[4] J. Camelio, S.J. Hu and D. Ceglarek: ASME J. Mech. Des. Vol. 125 (2003), p. 673

[5] S.C. Liu and S.J. Hu: ASME J. Manuf. Sci. Eng. Vol. 119 (1997), p. 368 